Article

\title{
Proteomic and Membrane Lipid Correlates of Re-duced Host Defense Peptide Susceptibility in a snoD Mutant of Staphylococcus aureus
}

\author{
Christian Kohler ${ }^{1,+}{ }^{+}$Richard A. Proctor ${ }^{2, \dagger}{ }^{\text {, Arnold S. Bayer }}{ }^{3,4}{ }^{\oplus}$, Michael R. Yeaman ${ }^{3,4,5}$, \\ Michael Lalk ${ }^{6}$, Susanne Engelmann ${ }^{1,7,8}$ and Nagendra N. Mishra ${ }^{3,4, *}$ \\ 1 Universität Greifswald, Institut für Mikrobiologie und Molekularbiologie,17487 Greifswald, Germany; \\ chritian.kohler@med.uni-greifswald.de (C.K.); susanne.engelmann@helmholtz-hzi.de (S.E.) \\ 2 University of Wisconsin School of Medicine and Public Health, Madison, WI 53705, USA; rap@wisc.edu \\ 3 Division of Infectious Diseases, Los Angeles Biomedical Research Institute at Harbor-UCLA Medical Center \\ Torrance, CA 90502, USA; abayer@labiomed.org (A.S.B); mryeaman@ucla.edu (M.R.Y.) \\ 4 David Geffen School of Medicine at UCLA, Los Angeles, CA 90095, USA \\ 5 Division of Molecular Medicine, Los Angeles Biomedical Research Institute at Harbor-UCLA Medical Center, \\ Torrance, CA 90502, USA \\ 6 University Greifswald, Institute of Biochemistry, 17487 Greifswald, Germany; lalk@uni-greifswald.de \\ 7 Institute for Microbiology, Technical University Braunschweig, Institute for Microbiology, 38106 \\ Braunschweig, Germany \\ 8 Helmholtz Center for Infectious Research, Microbial Proteomics, 38124 Braunschweig, Germany \\ * Correspondence: nnmishra@ucla.edu or nmishra@labiomed.org; Tel.: +310-222-4013; Fax: +310-803-5620 \\ + Contributed equally to the design of the study.
}

Received: 13 August 2019; Accepted: 24 September 2019; Published: 28 September 2019

check for updates

\begin{abstract}
We previously described a transposon mutant in Staphylococcus aureus strain SH1000 that exhibited reduced susceptibility to cationic thrombin-induced platelet microbicidal proteins (tPMPs). The transposon insertion site was mapped to the gene $s n o D$, the staphylococcal nuo orthologue. Hence, further studies have been performed to understand how this mutation impacts susceptibility to tPMP, by comparing proteomics profiling and membrane lipid analyses of the parent vs. mutant strains. Surprisingly, the mutant showed differential regulation of only a single protein when cultivated aerobically (FadB), and only a small number of proteins under anaerobic growth conditions (AdhE, DapE, Ddh, Ald1, IlvA1, AgrA, Rot, SA2366, and SA2367). Corresponding to FadB impact on lipid remodeling, membrane fatty acid analyses showed that the snoD mutant contained more short chain anteiso-, but fewer short chain iso-branched chain fatty acids under both aerobic and anaerobic conditions vs. the parental strain. Based upon these proteomic and membrane compositional data, a hypothetical "network" model was developed to explain the impact of the snoD mutation upon tPMP susceptibility.
\end{abstract}

Keywords: S. aureus; snoD mutant; lipids; proteomics; tPMP resistance

\section{Introduction}

Thrombin-induced platelet microbicidal proteins (tPMPs) represent an important component of innate immunity against endovascular infections, including those caused by both methicillin-susceptible and methicillin-resistant Staphylococcus aureus (MSSA; MRSA, respectively) [1]. For example, clinical S. aureus strains which exhibit reduced killing by low concentrations of tPMPs in vitro are associated with: i) endocarditis and vascular catheter-associated staphylococcemias (as compared to isolates from soft-tissue infections) [2]; ii) more prolonged bacteremia in patients with or without endocarditis [2,3]; 
and iii) increased persistence and/or reduced responsiveness to antibiotic treatment in humans [2] or experimental endocarditis [4-6]. Reduced susceptibility to tPMPs in vitro has also been correlated to a broad array of specific staphylococcal adaptive phenotypes: i) decreased membrane potential $(\Delta \psi)$ associated with interruption of either aerobic or anaerobic electron transport systems $[7,8]$; ii) increased surface positive charge due to enhanced lysinylation of phosphatidyl-glycerol (PG) and/or D-alanylation of wall teichoic acids [8]; iii) altered membrane order (i.e., extremes of fluidity or rigidity) [8-10]; iv) distinct changes in carotenoid content [9]; and v) alterations of the QacA membrane transporter [11].

Previously, we found that a transposon-induced mutant in the well-characterized MSSA strain, SH1000, was resistant to killing by low concentrations of tPMPs in vitro [4]. The transposon insert was mapped to a locus we termed snoD, for Staphylococcal $\underline{n}$ uo orthologue [12]. Phenotypically, in addition to reduced susceptibility to tPMPs, the mutation in snoD was associated with a reduced capacity to maintain $\Delta \psi$ over a standard growth cycle, as well as enhanced membrane fluidity [12].

Beyond snoD, previous attempts to delineate how specific genetic regulatory mechanism(s) and/or pathway(s) may be related to tPMP resistance have met with challenges. For example, our initial comparative gene expression profiles by microarray were not particularly revealing (unpublished), and suffered from several key limitations, especially: i) single growth phase time-point queries; and ii) aerobic growth conditions, given that the $s n o D$ operon might be most important in terms of anaerobic oxidative activities [12]. Therefore, to gain further insights into potential mechanism(s) behind the above phenotypes observed in the snoD mutant, we compared the proteomic and membrane fatty acid compositional profiles of this mutant with that of its isogenic parental strain, under aerobic as well as anaerobic conditions.

\section{Methods}

\subsection{Bacterial Strains and Growth Conditions}

The study strain-pair has been described in detail before [12]. In brief, MSSA parental strain, SH1000, and its isogenic snoD mutant were used in the present study. The snoD locus was insertionally inactivated by transposon mutagenesis [12], and the mutant designated as SH1000-98. Staphylococcal snoD, (like mrpD of Bacillus subtilis), is found in a seven-gene operon [13]. These genes encode homologous proteins, and the overall architecture of at least two operons is indistinguishable [13]. snoD encodes the unique gene product which is affected by the transposon mutation only [12]. The sno (mnh) operon was reported as $\mathrm{Na}+\mathrm{H}+$ antiporter function before [14]. As previously published, the SH-1000 parental strain is more susceptible to killing by sub-physiologic concentrations of tPMPs in vitro as compared to the snoD mutant, SH1000-98 (21 $\pm 10 \%$ vs. $59 \pm 2 \%$ survival after two hours exposure to $2 \mu \mathrm{g} / \mathrm{mL}$ tPMPs bioequivalent units, respectively; $p<0.05)$ [4]. This platelet peptide preparation has been previously shown to contain a mixture of tPMPs, wherein tPMP-1 is of quantitative predominance. In addition, the snoD mutant differs from the parental strain in the following parameters: i) cross-resistant to the membrane-active cationic peptide, protamine; and ii) enhanced membrane fluidity.

The bioactivity of the tPMP preparations was performed as described previously [15]. For protein preparation and lipid membrane analyses, $100 \mathrm{~mL}$ tryptic soy broth (TSB) (Oxoid, Wesel, Germany) was inoculated with exponentially growing cells of an overnight culture to an initial optical density at $540 \mathrm{~nm}\left(\mathrm{OD}_{540}\right)$ of 0.05 . Cells were cultivated under vigorous agitation at $37^{\circ} \mathrm{C}$ in baffled $500 \mathrm{~mL}$ Erlenmeyer flasks to an $\mathrm{OD}_{540}$ of 0.7 . Afterwards, $50 \mathrm{~mL}$ of the culture were shifted to anaerobic conditions by cultivating in $50 \mathrm{~mL}$ Falcon Tubes under gentle agitation at $37^{\circ} \mathrm{C}$. Anaerobic conditions were verified by using the redox indicator, resazurin, as previously described [16]. 


\subsection{Proteomic Analyses}

Cytoplasmic proteins of the parental strain and its isogenic snoD mutant were prepared in parallel from aerobically and anaerobically grown cells. Cells were harvested by centrifugation $\left(4{ }^{\circ} \mathrm{C}, 10 \mathrm{~min}\right.$ at $7000 \times g)$, and the resulting cell pellet was washed twice with ice-cold Tris-EDTA buffer. For cell lysis, cells were suspended in $1 \mathrm{~mL}$ ( $10 \mathrm{mM}$ Tris, $1 \mathrm{mM}$ EDTA, $1 \mathrm{mM}$ phenylmethylsulfonyl fluoride, $\mathrm{pH} 7.5$ ) and placed in screw-cap microtubes (Sarstedt, Germany) containing $500 \mu \mathrm{L}$ of glass beads (diameter 0.10 to 0.11 , Sartorius, Goettingen, Germany). Cells were mechanically disrupted as described [17]. The lysate was centrifuged twice at $21,000 \times g\left(4{ }^{\circ} \mathrm{C}\right)$ for 25 and $45 \mathrm{~min}$, respectively. The protein concentration of the supernatant was determined using Roti-Nanoquant (Roth, Karlsruhe, Germany) and protein solutions were stored at $-20^{\circ} \mathrm{C}$ until analyzed. To resolve the cytoplasmic proteins, 2-dimensional polyacrylamide gel electrophoresis (2D PAGE) was performed as described $[17,18]$. The proteins separated by 2D PAGE were stained with colloidal Coomassie brilliant blue [19] and the gels were scanned with a light scanner with an integrated transparency unit (Quatographic, Braunschweig, Germany). The 2D gels were then analyzed with the software Delta2D (Decodon $\mathrm{GmbH}$, Greifswald, Germany). To adjust for differences in the amount of cytoplasmic proteins detected on 2D gels of the parental vs. the mutant strain under aerobic, as well as anaerobic conditions, data sets obtained from three separate and independent replicates were used.

For identification of differentially expressed proteins, the respective spots were cut from the gels and digested with trypsin. The resulting peptides were analyzed by matrix-assisted laser desorption ionization-time of flight mass spectrometry (MALDI-TOF MS) as described [20,21] with several modifications detailed elsewhere [17]. The combined mass spectroscopy (MS) and MS/MS peak identity results were searched against the $S$. aureus 8325 protein data base with the Mascot search engine version 2.1.0.4. (Matrix Science; London, United Kingdom) using query parameters as published by Kohler et al. [17] where protein concentrations were considered different at $p<0.05$.

\subsection{Fatty Acid Extraction and Gas Chromatographic Analysis of Fatty Acid Methyl Esters (GC-FAME)}

To determine the membrane fatty acid composition, colonies of SH1000 and SH1000-98 were freshly sub-cultured from original plates and grown on sheep blood agar in a quadrant pattern at $35^{\circ} \mathrm{C}$, in parallel aerobically and anaerobically (in BBL Gaspacks, producing an atmosphere of more than $15 \% \mathrm{CO}_{2}$ ). A second subculture was streaked in order to fully acclimate the strains to these respective growth conditions. Approximately $20 \mathrm{mg}$ of cell mass were then harvested after 24 hours from the late exponential phase of growth (3Q) and placed into $13 \times 100 \mathrm{~mm}$ test tubes. Next, the cells were saponified, methylated, and fatty acid esters were extracted into hexane as described [9]. The resulting methyl ester mixtures were separated by an Agilent 5890 dual-tower gas chromatograph. Specifically, fatty acids were identified by a well-characterized microbial identification system, utilizing individual known fatty acids as positive controls (Sherlock 4.5; MIDI Inc.; Wilmington, DE). For statistical analysis, data were analyzed by the unpaired, two-tailed Student's $t$ test, with $p<0.05$ representing a significant difference. A minimum of two independent runs were performed on different days.

\subsection{Pathway Analysis and Bioinformatics}

Gene functions were imputed based on known genomic relationships available through the University of Nebraska Transposon Mutant Library (formerly, NARSA) [22]. Pathway analysis and interpretive results were derived in part using information obtained from the Kegg database [23].

\section{Results}

\subsection{Proteomics Analyses}

Under aerobic conditions, changes in the proteome of the $s n o D$ mutant were surprisingly quite limited (Table 1). Only SA0224 (similar to FadB = 3-hydroxyacyl-CoA dehydrogenase) was increased in the snoD mutant. This enzyme is involved in the $\beta$-oxidation of fatty acids, as well as in the propanoate 
and butanoate pathways. In contrast, under anaerobic conditions, more significant changes in the proteome of the snoD mutant were observed. For example, the mutant showed increased Ddh, a lactic acid dehydrogenase, and Ald1, an amino acid dehydratase. Both of these enzymes oxidize NADH as they perform their enzymatic roles. Additionally, IlvA1, an enzyme involved in deamination of amino acids, was also increased. This enzyme intersects pathways involved in the formation of branched chain fatty acids. Moreover, DapE, which metabolizes lysine to N-succinyl-LL-2,6-diaminopimelate, was decreased in the $s n o D$ mutant relative to its parental counterpart under anaerobic conditions. The reduction in DapE would be expected to increase the pool of lysine available for lysinylation of the cell membrane, thereby increasing the relative positive charge of the cell surface. Earlier publications suggested that snoD might be in an operon concerned primarily with osmoprotection, and that the snoD mutant was less halotolerant than its parental strain [12]. Therefore, proteomic analyses were performed aerobically and anaerobically, in parallel, after growth of the strains under several salt concentrations (range of $\mathrm{NaCl}$ concentrations $=1 \mathrm{M}, 2 \mathrm{M}, 2.5 \mathrm{M}$, and $3 \mathrm{M}$ ). No significant differences in proteome profiles, aerobically or anaerobically, were found in the $s n o D$ vs. parental strains when challenged across this range of high salt concentrations (data not shown).

Table 1. Proteins of which the amount was affected by snoD mutation under anaerobic conditions.

\begin{tabular}{ccc}
\hline Protein (Annotation From 315) & Function & Ratio log2 (SH1000/snoD) \\
\hline Increased in parent & & \\
AdhE (SA0143) & Acetaldehyde DH & 1.46 \\
(SA2367) & Theoretical protein & 1.044 \\
\hline Decreased in mutant & Lysine metabolism & 1.021 \\
DapE (SA1814) & Theoretical protein & 1.051 \\
(SA2366) & Virulence regulator & 1.287 \\
AgrA (SA1844) & & \\
Increased in mutant & Threonine/serine dehydrase & -4.17 \\
IlvA1 (SA1271) & $\alpha$-amino acid dehydrogenase & -1.667 \\
Ald1 (SA1272) & D-lactate dehydrogenase & -1.006 \\
Ddh (SA2312) &
\end{tabular}

\subsection{Fatty Acid Analyses}

Bacterial growth entails a substantial portion of the acyl chains of lipids in membrane to maintain the optimum level of fluidity/rigidity $[9,10,24]$. Previous studies have shown that changes in the fatty acid composition of $S$. aureus can correlate with in vitro resistance to cationic host defense peptides, including tPMPs $[9,10]$. Hence, we examined fatty acid compositions of the parent and snoD mutant strains under both aerobic and anaerobic conditions. Among fatty acid species prevalent in S. aureus, large changes in membrane fluidity begin to occur at $\mathrm{C} 17$, and are even more pronounced at C15 and C13; hence, these species were grouped in this manner for our analyses. As can be seen in Table 2, both parental and mutant strains showed major fatty acid content differences anaerobically, with significantly decreased anteiso-branched chain, unsaturated-, and polyunsaturated-fatty acids. Also, the amount of short iso-branched fatty acids increased, while long iso-branched fatty acids decreased with a shift to anaerobic conditions. The snoD mutant contained more short chain anteiso-branched fatty acids and fewer short chain iso-branched fatty acids under both growth conditions as compared to the parental strain. In contrast, unsaturated fatty acid profiles were increased to an equivalent extent in both of the strains under anaerobic conditions (full fatty acid analysis shown as Table 3). 
Table 2. Summary fatty acid compositional differences between parent and snoD mutant.

\begin{tabular}{|c|c|c|c|c|c|c|}
\hline \multirow{2}{*}{$\begin{array}{l}\text { Fatty Acid } \\
\text { Species }\end{array}$} & \multicolumn{2}{|c|}{ SH1000-98 } & \multirow{2}{*}{${ }^{*} p$ value } & \multicolumn{2}{|c|}{ SH1000-WT } & \multirow{2}{*}{${ }^{*} p$ value } \\
\hline & Aerobic & Anaerobic & & Aerobic & Anaerobic & \\
\hline $\begin{array}{l}\text { Anteiso } \\
\text { (C13-17) }\end{array}$ & $64.49 \pm 0.11$ & $39.07 \pm 0.49$ & 0.006 & $61.16 \pm 1.16$ & $35.85 \pm 1.10$ & 0.002 \\
\hline $\begin{array}{l}\text { Unsaturated } \\
\text { Polyunsaturated } \\
\text { subset (C20) }\end{array}$ & $\begin{array}{l}3.58 \pm 0.14 \\
0.42 \pm 0.01\end{array}$ & $\begin{array}{c}12.81 \pm 0.16 \\
0.49 \pm 0.01\end{array}$ & $\begin{array}{c}0.0003 \\
0.045\end{array}$ & $\begin{array}{l}3.565 \pm 0.40 \\
0.315 \pm 0.01\end{array}$ & $\begin{array}{c}12.10 \pm 0.09 \\
0.42 \pm 0.01\end{array}$ & $\begin{array}{l}0.016 \\
0.027\end{array}$ \\
\hline $\begin{array}{c}\text { Short Isos } \\
\text { (C13-15) }\end{array}$ & $5.97 \pm 0.08$ & $14.69 \pm 0.25$ & 0.007 & $6.895 \pm 0.13$ & $16.60 \pm 0.41$ & 0.015 \\
\hline $\begin{array}{l}\text { Long Isos } \\
\text { (C16-20) }\end{array}$ & $6.03 \pm 0.05$ & $5.205 \pm 0.05$ & 0.003 & $7.195 \pm 0.08$ & $5.30 \pm 0.03$ & 0.009 \\
\hline
\end{tabular}

$* p$-value $=$ aerobic vs. anaerobic.

Table 3. Complete fatty acid analysis of strain-pair under aerobic and anaerobic conditions.

\begin{tabular}{|c|c|c|c|c|c|c|}
\hline \multirow{2}{*}{$\begin{array}{l}\text { Fatty Acid } \\
\text { Species }\end{array}$} & \multicolumn{2}{|c|}{ Aerobic } & \multirow[b]{2}{*}{$p$-value * } & \multicolumn{3}{|c|}{ Anaerobic } \\
\hline & SH1000-WT & SH1000-98 & & SH1000-WT & SH1000-98 & $p$-value ${ }^{* *}$ \\
\hline $13: 0$ iso & - & & & $0.325 \pm 0.01$ & $0.265 \pm 0.02$ & \\
\hline 13:0 anteiso & - & $0.105 \pm 0.01$ & & - & - & \\
\hline $14: 0$ iso & $1.18 \pm 0.03$ & $0.78 \pm 0.01$ & 0.01 & $1.985 \pm 0.02$ & $1.455 \pm 0.04$ & 0.007 \\
\hline 14:0 & $0.215 \pm 0.02$ & $0.195 \pm 0.01$ & & $1.07 \pm 0.08$ & $0.765 \pm 0.011$ & \\
\hline $15: 1$ iso $G$ & $0.165 \pm 0.01$ & $0.15 \pm 0.00$ & & $0.325 \pm 0.02$ & - & \\
\hline $15: 0$ iso & $5.75 \pm 0.11$ & $5.185 \pm 0.06$ & & $14.29 \pm 0.42$ & $12.97 \pm 0.20$ & \\
\hline 15:0anteiso & $43.96 \pm 0.72$ & $46.15 \pm 0.04$ & & $31.025 \pm 0.93$ & $33.495 \pm 0.40$ & \\
\hline 15:0 & - & - & & $0.795 \pm 0.05$ & $0.31 \pm 0.01$ & 0.03 \\
\hline $16: 0$ iso & $3.05 \pm 0.09$ & $1.965 \pm 0.06$ & 0.009 & $1.17 \pm 0.01$ & $1.11 \pm 0.01$ & \\
\hline 16:0 & $1.75 \pm 0.10$ & $1.81 \pm 0.00$ & & $3.94 \pm 0.16$ & $3.98 \pm 0.06$ & \\
\hline $15: 02 \mathrm{OH}$ & - & - & & $0.23 \pm 0.01$ & $0.205 \pm 0.01$ & \\
\hline $17: 0$ iso & $3.93 \pm 0.07$ & $3.55 \pm 0.06$ & & $3.835 \pm 0.01$ & $3.695 \pm 0.05$ & \\
\hline $17: 0$ anteiso & $17.2 \pm 0.44$ & $18.24 \pm 0.16$ & & $4.82 \pm 0.17$ & $5.57 \pm 0.08$ & 0.06 \\
\hline $17: 0$ & $0.215 \pm 0.04$ & $0.2 \pm 0.01$ & & $2.075 \pm 0.011$ & $0.96 \pm 0.04$ & 0.02 \\
\hline $18: 0$ iso & $1.555 \pm 0.01$ & $1.02 \pm 0.01$ & 0.0025 & $0.395 \pm 0.04$ & $0.375 \pm 0.04$ & \\
\hline 18:1w9c & $2.775 \pm 0.04$ & $3 \pm 0.13$ & & $5.91 \pm 0.03$ & $6.13 \pm 0.08$ & \\
\hline 18:1w7C & $0.525 \pm 0.01$ & $0.58 \pm 0.01$ & & $1.35 \pm 0.03$ & $1.515 \pm 0.04$ & \\
\hline 18:0 & $6.75 \pm 0.014$ & $6.93 \pm 0.08$ & & $10.4 \pm 0.17$ & $10.58 \pm 0.00$ & \\
\hline $19: 0$ iso & $1.335 \pm 0.01$ & $1.185 \pm 0.01$ & & $1.07 \pm 0.01$ & $1.135 \pm 0.04$ & \\
\hline 19:0 anteiso & $3.61 \pm 0.04$ & $3.68 \pm 0.03$ & & $0.97 \pm 0.04$ & $1.225 \pm 0.02$ & 0.04 \\
\hline 19:0 & $0.245 \pm 0.04$ & $0.235 \pm 0.01$ & & $2.25 \pm 0.18$ & $1.145 \pm 0.06$ & \\
\hline $20: 4$ & $0.315 \pm 0.01$ & $0.415 \pm 0.01$ & 0.005 & $0.42 \pm 0.01$ & $0.49 \pm 0.01$ & 0.04 \\
\hline \multicolumn{7}{|l|}{$w 6,9,12,15 c$} \\
\hline $20: 1 \mathrm{w} 9 \mathrm{c}$ & 2.49 & - & & $4.835 \pm 0.04$ & $5.165 \pm 0.04$ & \\
\hline 20:0 & $2.5 \pm 0.056$ & $2.615 \pm 0.063$ & & $3.495 \pm 0.08$ & $4.055 \pm 0.06$ & 0.02 \\
\hline $20: 0$ iso & $0.375 \pm 0.01$ & $0.31 \pm 0.014$ & & - & - & \\
\hline
\end{tabular}

${ }^{*} p$-value $=$ comparison between aerobic condition; ${ }^{* *} p$-value $=$ comparison between anaerobic condition.

\section{Discussion}

The scope of significant proteomic changes in the $s n o D$ mutant strain was surprisingly limited, especially under aerobic conditions. In particular, only locus SA0224 (similar to FadB = 3-hydroxyacyl-CoA dehydrogenase) was differentially upregulated in the $s n o D$ mutant under aerobic conditions. FadB shortens fatty acid chains, which would be consistent with the previously documented increases in membrane fluidity in our snoD mutant [12]. In turn, this observation is consistent with prior linkage of increased membrane fluidity phenotypes with increased resistance of $S$. aureus to tPMPs in vitro $[1,7-10,25]$. Increased membrane fluidity is associated with proton leak and reduced membrane 
potential [12] with increased tPMP resistance, as shown in the model (Figure 1). As illustrated, $\beta$-oxidation of fatty acids by FadB yields NADH, which must then be oxidized for metabolism to continue, as a large number of dehydrogenases require this substrate as a co-factor. Because the cytochrome oxidase system would be fully functional aerobically, the products of central metabolism that generate NADH could be efficiently processed under aerobic conditions by cytochrome oxidase. Importantly, Sharma-Kuinkel et al. previously demonstrated that the Fad operon correlates with clonal complex type 30 (CC30) and may confer upon S. aureus the ability to adapt for survival in the face of cationic agents such as daptomycin [26]. In turn, the Fad operon may give staphylococcal strains virulence advantages in persistent or other human infections.

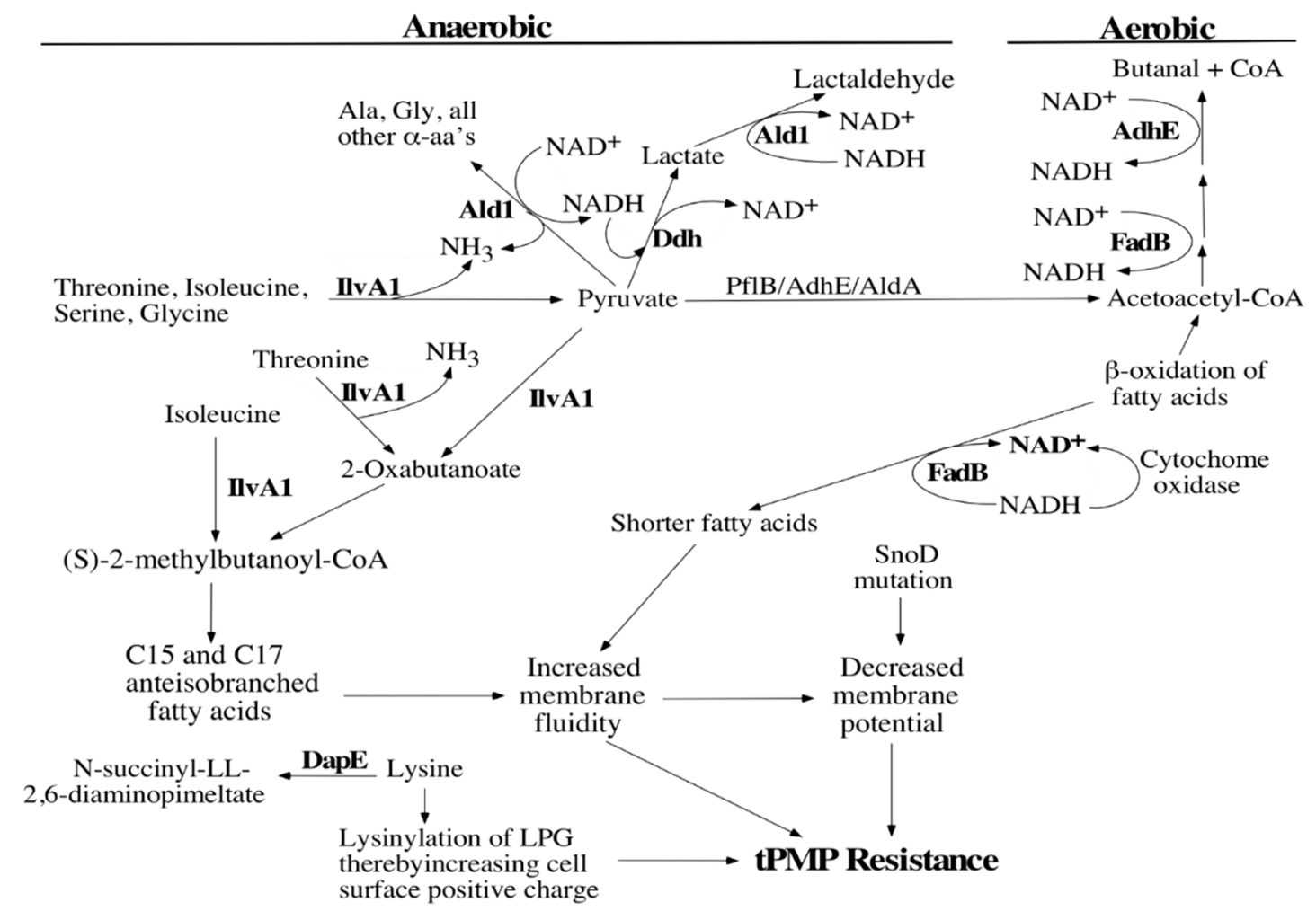

Figure 1. Integrative hypothetical model of reduced tPMP susceptibility in S. aureus. This hypothetical model was derived from bioinformatics analysis and incorporates the data presented in this paper and previous work. This model illustrates how $S$. aureus can adapt to a phenotype that has reduced susceptibility to $\mathrm{tPMP}$ with a single mutation in $s n o D$. Abbreviations used in figure: AdhE = alcohol-acetaldehyde dehydrogenase; Ald1 = $\alpha$-amino acid dehydrogenase; DapE = succinyl-diaminopimelate desuccinylase; Ddh = D-lactate dehydrogenase; FadB = 3-hydroxyacyl-CoA dehydrogenase; IlvA1 = Threonine/serine dehydrase; $\mathrm{PflB}=$ formate acetyltransferase; $s n o D=$ S. aureus nuo orthologue; $\mathrm{PMP}=$ thrombin-induced platelet microbicidal protein.

More notable changes were identified in the proteome of the snoD mutant under anaerobic vs. anaerobic conditions, which is consistent with our previous findings, wherein differences in tPMP susceptibility were greatest under microaerophilic conditions [12]. While none of the proteins in the sno operon have NADH oxidase activity, these proteins may be linked to the upstream protein, Ndh2 oxidase, and the membrane potential-forming protein, MspA [27]. In any event, a consistent pattern is seen in the proteins induced under anaerobic conditions; i.e., these proteins are clearly linked to NADH oxidation. For example, the Ddh protein in S. aureus serves as a NAD ${ }^{+}$-dependent lactate dehydrogenase [28]. We found Ddh to be significantly upregulated in the snoD mutant under anaerobic conditions. Likewise, the Ald1 protein is over-represented anaerobically in the snoD mutant proteome as compared to its isogenic parental strain. Importantly, we previously reported that Ald1 expression 
aerobically was increased in the tPMP-resistant hemB and menD small colony variants, both of which have defects in oxidizing NADH $[15,29]$.

As a unifying model, we have related the observed changes in Ddh and Ald1 profiles in the snoD mutant to the relative redox state, wherein pathways optimized to oxidize NADH anaerobically are likely operative (Figure 1). The relatively greater increase in AdhE in the parent probably relates to the fact that this protein may already be adequately expressed in the mutant, but needs to be induced in the parent to accomplish anaerobic oxidation of NADH.

In addition to changes in NADH/NAD ${ }^{+}$cycling in the snoD mutant, we identified protein perturbations that should facilitate changes in cell membrane lipid composition. In S. aureus, the IlvA1 protein acts as a threonine desaturase, which catalyzes the anaerobic formation of 2-oxobutanoate ( $\alpha$-ketobutyrate) and ammonia from threonine in a two-step reaction (Figure 1). IlvA1 shuttles amino acids towards (S)-2-methylbutanoyl-CoA, which would be expected to increase both the proportion of C15 and C17 anteiso-branched fatty acids and, consequently, the membrane fluidity. The ratio of anteiso- to short iso-branch chain lipids is approximately 2.6 in the snoD mutant, as compared to $2.1 \mathrm{in}$ the parent. This shift in the lipid profile of the $s n o D$ mutant strain is similar to the membrane perturbations in B. subtilis during "cold adaptation" under anaerobic conditions [24], a modification that also increases membrane fluidity [30]. Anteiso-branched fatty acids produce more fluid membranes as compared to iso-branched chain fatty acids [31]); hence, the present results support the previously identified changes in membrane fluidity in the snoD mutant [10]. The "breakpoint" for changes in membrane fluidity occurs in iso-branched chain fatty acids at C15-to-C16 species. Thus, we used this breakpoint to group our data for relevant analyses. While the amount of anteiso-branched fatty acids is normally lower anaerobically (which would hypothetically render cell membranes more rigid), the anteiso-branched fatty acids in the snoD mutant are maintained at relatively higher levels, thereby allowing for greater fluidity than the parent. All of the changes in fatty acid length and branching favor increased membrane fluidity.

In theory, increased membrane fluidity may impact susceptibility to tPMPs and other cationic host defense peptides in at least two ways. First, the insertion capacity of these cationic peptides into target membranes is likely to be physically altered by such extremes of membrane order $[1,7-10,25,32]$. Second, a more fluid membrane allows for greater transmembrane leakage of protons, dissipating the $\Delta \psi$ [33], a phenotype that is critically involved in tPMP activity [7]. In direct support of this notion, the mutation in $s n o D$ is associated with a substantial decrease in $\Delta \psi$ [12]. One possible explanation for this observation is that increased fluidity would allow fewer tPMP molecules to insert into the cell membrane of the snoD mutant, limiting their membrane perturbing actions. This defect would also blunt their eventual penetration into the cytoplasmic compartment of the snoD mutant where these peptides are believed to exert multiple antimicrobial mechanisms [9-12,34]. Finally, reduced DapE content in the $s n o D$ mutant would decrease lysine breakdown (Figure 1). This would increase lysine availability for lysinylating phosphatidylglycerol (PG) and synthesizing this positively-charged membrane species, lysyl-PG; this event, in turn, would likely increase the relative positive surface charge in the snoD mutant, resulting in a "charge-repulsive milieu" against host cationic peptides, like tPMPs [8].

In summary, we have previously reported that mutation and loss-of-function of the SnoD complex in S. aureus is correlated with increased membrane fluidity and reduced $\Delta \psi$, as well as with decreased NADH oxidation and increased tPMP resistance. This mutant appears to foster these adaptations by engaging alternative pathways to oxidize $\mathrm{NADH}$, increase membrane fluidity, and redirect metabolism to allow for a relative increase in surface charge. Of most importance, by modifying the expression of only a very small group of proteins, the organism becomes more resistant to an important host defense peptide (tPMP), supporting its survivability under stress conditions. 
Author Contributions: Investigation, C.K.; methodology, M.L.; supervision, investigation, writing and review; N.N.M.; supervision, writing-original draft, R.A.P.; supervision, writing, review and editing, A.S.B., M.R.Y. and S.E.

Funding: National Institutes of Health: AI-39108; National Institutes of Health: U01-AI-124319 and R33-AI-111661; Deutsche Forschungsgemeinschaft TRR34 and INST 188/365-1 FUGG. LA Biomed at Harbor-UCLA Medical Center, Torrance, CA.: 531604-01-00.

Acknowledgments: This research was supported in part by grants AI-39108 (to Arnold S. Bayer) and U01-AI-124319 and R33-AI-111661 (to Michael R. Yeaman) from the U.S. National Institutes of Health and the Deutsche Forschungsgemeinschaft TRR34 and INST 188/365-1 FUGG (to Susanne Engelmann) NNM was supported by an intramural grant from the LA Biomed-Harbor UCLA Medical Center, Torrance, CA.

Conflicts of Interest: Michael R. Yeaman is founder of NovaDigm Therapeutics, Inc. which develops novel anti-infective agents, vaccines and immunotherapies. The other authors declare no conflict of interest.

\section{References}

1. Yeaman, M.R.; Bayer, A.S. Antimicrobial peptides from platelets. Drug Resist. Updates 1999, 2, $116-126$. [CrossRef] [PubMed]

2. Fowler, V.G.J.; Sakoulas, G.; McIntyre, L.M.; Meka, V.G.; Arbeit, R.D.; Cabell, C.H.; Stryjewski, M.E.; Eliopoulos, G.M.; Reller, L.B.; Corey, G.R.; et al. Persistent bacteremia due to methicillin-resistant Staphylococcus aureus infection is associated with agr dysfunction and low-level in vitro resistance to thrombin-induced platelet microbicidal protein. J. Infect. Dis. 2004, 190, 1140-1149. [CrossRef] [PubMed]

3. Moise, P.A.; Forrest, A.; Bayer, A.S.; Xiong, Y.Q.; Yeaman, M.R.; Sakoulas, G. Factors influencing time to vancomycin-induced clearance of nonendocarditis methicillin-resistant Staphylococcus aureus bacteremia: Role of platelet microbicidal protein killing and agr genotypes. J. Infect. Dis. 2010, 201, 233-240. [CrossRef] [PubMed]

4. Dhawan, V.; Yeaman, M.R.; Kim, E.; Cheung, A.L.; Sullam, P.M.; Bayer, A.S. Phenotypic resistance to thrombin-induced platelet microbicidal protein in vitro correlates with enhanced virulence in experimental endocarditis due to Staphylococcus aureus. Infect. Immun. 1997, 65, 3293-3299. [PubMed]

5. Kupferwasser, L.I.; Yeaman, M.R.; Shapiro, S.M.; Nast, C.C.; Bayer, A.S. In vitro susceptibility to thrombin-induced platelet microbicidal protein is associated with reduced disease progression and complication rates in experimental Staphylococcus aureus endocarditis: Microbiological, histopathologic, and echocardiographic analyses. Circulation 2002, 105, 746-752. [PubMed]

6. Mercier, R.C.; Dietz, R.M.; Mazzola, J.L.; Bayer, A.S.; Yeaman, M.R. Beneficial influence of platelets on antibiotic efficacy in an in vitro model of Staphylococcus aureus-induced endocarditis. Antimicrob. Agents Chemother. 2004, 48, 2551-2557. [CrossRef] [PubMed]

7. Koo, S.P.; Bayer, A.S.; Sahl, H.G.; Proctor, R.A.; Yeaman, M.R. Staphylocidal action of thrombin-induced platelet microbicidal protein is not solely dependent on transmembrane potential. Infect. Immun. 1996, 64, 1070-1074. [PubMed]

8. Weidenmaier, C.; Peschel, A.; Kempf, V.A.; Lucindo, N.; Yeaman, M.R.; Bayer, A.S. DltABCD-and MprF-mediated cell envelope modifications of Staphylococcus aureus confer resistance to platelet microbicidal proteins and contribute to virulence in a rabbit endocarditis model. Infect. Immun. 2005, 73, 8033-8038. [CrossRef] [PubMed]

9. Mishra, N.N.; Liu, G.Y.; Yeaman, M.R.; Nast, C.C.; Proctor, R.A.; McKinnell, J.; Bayer, A.S. Carotenoid-related alteration of cell membrane fluidity impacts Staphylococcus aureus susceptibility to host defense peptides. Antimicrob. Agents Chemother. 2011, 55, 526-531. [CrossRef] [PubMed]

10. Bayer, A.S.; Prasad, R.; Chandra, J.; Koul, A.; Mishra, S.; Varma, A.; Skurray, R.A.; Firth, N.; Brown, M.H.; Koo, S.P.; et al. In vitro resistance of Staphylococcus aureus to thrombin-induced platelet microbicidal protein is associated with alterations in cytoplasmic membrane fluidity. Infect. Immun. 2000, 68, 3548-3553. [CrossRef] [PubMed]

11. Bayer, A.S.; Kupferwasser, L.I.; Brown, M.H.; Skurray, R.A.; Grkovic, S.; Jones, T.; Mukhopadhay, K.; Yeaman, M.R. Low-level resistance of Staphylococcus aureus to thrombin-induced platelet microbicidal protein-1 in vitro associated with qacA gene carriage is independent of multidrug efflux pump activity. Antimicrob. Agents Chemother. 2006, 50, 2448-2454. [CrossRef] [PubMed] 
12. Bayer, A.S.; McNamara, P.; Yeaman, M.R.; Lucindo, N.; Jones, T.; Cheung, A.L.; Sahl, H.G.; Proctor, R.A. Transposon disruption of the complex I NADH oxidoreductase gene (snoD) in Staphylococcus aureus is associated with reduced susceptibility to the microbicidal activity of thrombin-induced platelet microbicidal protein-1. J. Bacteriol. 2006, 188, 211-222. [CrossRef] [PubMed]

13. Ito, M.; Guffanti, A.A.; Oudegam, B.; Krulwich, T.A. Mrp, a multigene, multifunctional locus in Bacillus subtilis with roles in resistance to cholate and to $\mathrm{Na}^{+}$and in $\mathrm{pH}$ homeostasis. J. Bacteriol. 1999, 181, $2394-2402$. [PubMed]

14. Hiramatsu, T.; Kodama, K.; Kuroda, T.; Mizushima, T.; Tsuchiya, T. A putative multisubunit $\mathrm{Na}^{+} / \mathrm{H}^{+}$ antiporter from Staphylococcus aureus. J. Bacteriol. 1998, 180, 6642-6648. [PubMed]

15. Yeaman, M.R.; Puentes, S.M.; Norman, D.C.; Bayer, A.S. Partial purification and staphylocidal activity of thrombin-induced platelet microbicidal protein. Infect. Immun. 1992, 60, 1202-1209. [PubMed]

16. Fuchs, S.; Pané-Farré, J.; Kohler, C.; Hecker, M.; Engelmann, S. Anaerobic gene expression in Staphylococcus aureus. J. Bacteriol. 2007, 189, 4275-4289. [CrossRef] [PubMed]

17. Kohler, C.; von Eiff, C.; Liebeke, M.; McNamara, P.J.; Lalk, M.; Proctor, R.A.; Hecker, M.; Engelmann, S. A defect in menadione biosynthesis induces global changes in gene expression in Staphylococcus aureus. J. Bacteriol. 2008, 190, 6351-6364. [CrossRef] [PubMed]

18. Büttner, K.; Bernhardt, J.; Scharf, C. A comprehensive two-dimensional map of cytosolic proteins of Bacillus subtilis. Electrophoresis 2001, 22, 2908-2935. [CrossRef]

19. Engelmann, S.; Hecker, M. Proteomic analysis to investigate regulatory networks in Staphylococcus aureus. Methods Mol. Biol. 2008, 431, 25-45.

20. Candiano, G.; Bruschi, M.; Musante, L.; Santucci, L.; Ghiggeri, G.M.; Carnemolla, B.; Orecchia, P.; Zardi, L.; Righetti, P.G. Blue silver: A very sensitive colloidal coomassie G-250 staining for proteome analysis. Electrophoresis 2004, 25, 1327-1333. [CrossRef]

21. Eymann, C.; Dreisbach, A.; Albrecht, D.; Gentner, S.; Tam, L.T.; Büttner, K.; Buurman, G.; Scharf, C.; Venz, S.; Völker, U.; et al. A comprehensive proteome map of growing Bacillus subtilis cells. Proteomics 2004, 4, 2849-2876. [CrossRef]

22. University of Nebraska Transposon Mutant Library, University of Nebraska Medical Center, Department of Pathology \& Microbiology Center for Staphylococcal Research [CSR]. 2019. Available online: www.unmc. edu/pathology/csr/research/library.html (accessed on 10 June 2019).

23. Kegg Pathway Maps. Kanehisa Laboratories, Japan. 2019. Available online: www.genome.jp/kegg/kegg3a. html (accessed on 10 June 2019).

24. Beranová, J.; Mansilla, M.C.; de Mendoza, D.; Elhottová, D.; Konopásek, I. Differences in cold adaptation of Bacillus subtilis under anaerobic and aerobic conditions. J. Bacteriol. 2010, 192, 4164-4171. [CrossRef] [PubMed]

25. Mukhopadhyay, K.; Whitmire, W.; Xiong, Y.Q.; Molden, J.; Jones, T.; Peschel, A.; Staubitz, P.; Adler-Moore, J.; McNamara, P.J.; Proctor, R.A.; et al. In vitro susceptibility of Staphylococcus aureus to thrombin-induced platelet microbicidal protein-1 (tPMP-1) is influenced by cell membrane phospholipid composition and asymmetry. Microbiology 2007, 153, 1187-1197. [CrossRef] [PubMed]

26. Sharma-Kuinkel, B.K.; Mongodin, E.F.; Myers, J.R.; Vore, K.L.; Canfield, G.S.; Fraser, C.M.; Rude, T.H.; Fowler, V.G., Jr.; Gill, S.R. Potential Influence of Staphylococcus aureus clonal complex 30 genotype and transcriptome on hematogenous infections. Open Forum Infect. Dis. 2015, 2, ofv093. [CrossRef] [PubMed]

27. Mayer, S.; Steffen, W.; Steuber, J.; Götz, F. The Staphylococcus aureus Nuo-like protein MpsA, contributes to the generation of membrane potential. J. Bacteriol. 2015, 197, 794-806. [CrossRef] [PubMed]

28. Boyle-Vavra, S.; de Jonge, B.L.; Ebert, C.C.; Daum, R.S. Cloning of the Staphylococcus aureus ddh gene encoding $\mathrm{NAD}^{+}$-dependent D-lactate dehydrogenase and insertional inactivation in a glycopeptide-resistant isolate. J. Bacteriol. 1997, 179, 6756-6763. [CrossRef] [PubMed]

29. Kohler, C.; von Eiff, C.; Peters, G.; Proctor, R.A.; Hecker, M.; Engelmann, S. Physiological characterization of a heme- deficient mutant of Staphylococcus aureus by a proteomic approach. J. Bacteriol. 2003, 185, 6928-6937. [CrossRef]

30. Klein, W.; Weber, M.H.; Marahiel, M.A. Cold shock response of Bacillus subtilis: Isoleucine-dependent switch in the fatty acid branching pattern for membrane adaptation to low temperatures. J. Bacteriol. 1999, 181, 5341-5349. [PubMed] 
31. Kaneda, T. Iso-and anteiso-fatty acids in bacteria: Biosynthesis, function, and taxonomic significance. Microbiol. Rev. 1991, 55, 288-302.

32. Yount, N.Y.; Cohen, S.E.; Kupferwasser, D.; Waring, A.J.; Ruchala, P.; Sharma, S.; Wasserman, K.; Jung, C.-L.; Yeaman, M.R. Context mediates antimicrobial efficacy of kinocidin congener peptide RP-1. PLoS ONE 2011, 6, e26727. [CrossRef]

33. Haines, T.H. Do sterols reduce proton and sodium leaks through lipid bilayers? Prog. Lipid Res. 2001, 40, 299-324. [CrossRef]

34. Xiong, Y.Q.; Bayer, A.S.; Elazegui, L.; Yeaman, M.R. A synthetic congener modeled on a microbicidal domain of thrombin-induced platelet microbicidal protein-1 recapitulates staphylocidal mechanisms of the native molecule. Antimicrob. Agents Chemother. 2006, 50, 3786-3792. [CrossRef] [PubMed]

(C) 2019 by the authors. Licensee MDPI, Basel, Switzerland. This article is an open access article distributed under the terms and conditions of the Creative Commons Attribution (CC BY) license (http://creativecommons.org/licenses/by/4.0/). 